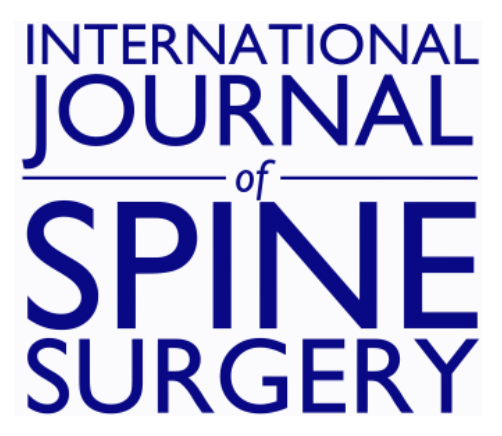

\title{
Anterior and Lateral Lumbar Interbody Fusion With Supplemental Interspinous Process Fixation: Outcomes from a Multicenter, Prospective, Randomized, Controlled Study
}

RIPUL PANCHAL, RYAN DENHAESE, CLINT HILL, K. BRANDON STRENGE, ALEXANDRE DE MOURA, PETER PASSIAS, PAUL ARNOLD, ANDREW CAPPUCCINO, M. DAVID DENNIS, ANDY KRANENBURG, BRIETA VENTIMIGLIA, KIM MARTIN, CHRIS FERRY, SARAH MARTINECK, CAMILLE MOORE and KEE KIM

Int J Spine Surg 2018, 12 (2) 172-184

doi: https://doi.org/10.14444/5025

http://ijssurgery.com/content/12/2/172

This information is current as of April 25, 2023.

Email Alerts Receive free email-alerts when new articles cite this article. Sign up at: http://ijssurgery.com/alerts 


\title{
Anterior and Lateral Lumbar Interbody Fusion With Supplemental Interspinous Process Fixation: Outcomes from a Multicenter, Prospective, Randomized, Controlled Study
}

\author{
RIPUL PANCHAL, DO, ${ }^{1}$ RYAN DENHAESE, MS, $\mathrm{MD},{ }^{2}$ CLINT HILL, $\mathrm{MD},{ }^{3} \mathrm{~K}$. BRANDON STRENGE, MD ${ }^{3}$ \\ ALEXANDRE DE MOURA, MD,${ }^{4}$ PETER PASSIAS, MD,${ }^{4}$ PAUL ARNOLD, MD,${ }^{5}$ ANDREW CAPPUCCINO, \\ MD ${ }^{6} \mathrm{M}$. DAVID DENNIS, MD,${ }^{7}$ ANDY KRANENBURG, $M D,{ }^{8}$ BRIETA VENTIMIGLIA, MS, ${ }^{9} \mathrm{KIM}$ \\ MARTIN, MS, PhD,${ }^{9}$ CHRIS FERRY, MS, ${ }^{9}$ SARAH MARTINECK, PA, ${ }^{10}$ CAMILLE MOORE, PhD,${ }^{11}$ KEE \\ $\mathrm{KIM}, \mathrm{MD}^{1}$ \\ ${ }^{1}$ University of California Davis Health System, Sacramento, California, ${ }^{2}$ AXIS Neurosurgery and Spine, Williamsville, New York, ${ }^{3}$ The Orthopaedic Institute, \\ Paducah, Kentucky, ${ }^{4}$ New York Spine Institute, Westbury, New York, ${ }^{5}$ Department of Neurosurgery, University of Kansas, Kansas City, Kansas, ${ }^{6}$ Buffalo Spine \\ Surgery, Lockport, New York, ${ }^{7}$ South Texas Spinal Clinic, Laredo, Texas, ${ }^{8}$ Southern Oregon Orthopaedics, Medford, Oregon, ${ }^{9}$ Zimmer Biomet Spine, \\ Broomfield, Colorado, ${ }^{10}$ RPA Consulting, Grand Island, New York, ${ }^{11}$ University of Colorado-Denver, Denver, Colorado
}

\begin{abstract}
Background: Rigid interspinous process fixation (ISPF) has received consideration as an efficient, minimally disruptive technique in supporting lumbar interbody fusion. However, despite advantageous intraoperative utility, limited evidence exists characterizing midterm to long-term clinical outcomes with ISPF. The objective of this multicenter study was to prospectively assess patients receiving single-level anterior (ALIF) or lateral (LLIF) lumbar interbody fusion with adjunctive ISPF.

Methods: This was a prospective, randomized, multicenter (11 investigators), noninferiority trial. All patients received single-level ALIF or LLIF with supplemental ISPF $(n=66)$ or pedicle screw fixation (PSF; $n=37)$ for degenerative disc disease and/or spondylolisthesis (grade $\leq 2$ ). The randomization patient ratio was 2:1, ISPF/PSF. Perioperative and follow-up outcomes were collected (6 weeks, 3 months, 6 months, and 12 months).

Results: For ISPF patients, mean posterior intraoperative outcomes were: blood loss, $70.9 \mathrm{~mL}$; operating time, 52.2 minutes; incision length, $5.5 \mathrm{~cm}$; and fluoroscopic imaging time, 10.4 seconds. Statistically significant improvement in patient Oswestry Disability Index scores were achieved by just 6 weeks after operation $(P<.01)$ and improved out to 12 months for the ISPF cohort. Patient-reported 36-Item Short Form Health Survey and Zurich Claudication Questionnaire scores were also significantly improved from baseline to 12 months in the ISPF cohort $(P<.01)$. A total of $92.7 \%$ of ISPF patients exhibited interspinous fusion at 12 months. One ISPF patient $(1.5 \%)$ required a secondary surgical intervention of possible relation to the posterior instrumentation/ procedure.

Conclusion: ISPF can be achieved quickly, with minimal tissue disruption and complication. In supplementing ALIF and LLIF, ISPF supported significant improvement in early postoperative ( $\leq 12$ months) patient-reported outcomes, while facilitating robust posterior fusion.
\end{abstract}

Lumbar Spine

Keywords: interspinous process fixation, ISPF, lateral lumbar interbody fusion, LLIF, anterior lumbar interbody fusion, ALIF, degenerative disc disease, lumbar spine, posterior fixation, minimally invasive, MIS, spondylolisthesis

\section{INTRODUCTION}

Anterior (ALIF) and lateral (LLIF) lumbar interbody fusion techniques combined with pedicle screw and rod fixation (PSF) are widely accepted surgical strategies to treat pain, deformity, and instability associated with degenerative spine disease. ${ }^{1-12}$ However, given the inherent axial and coronal stability of a large anterolateral cage, the structural necessity of invasive/extensive PSF in ALIF/LLIF has come into question, particularly in single-level application. Accordingly, a growing body of literature exploring less invasive and less demanding alternatives for secondary stability in ALIF/LLIF has emerged. ${ }^{13-31}$ The ability to provide effective rigidity in support of arthrodesis while 
avoiding the risks associated with PSF-which include neural, vascular, and dural injury, and accelerated adjacent segment pathology-would prove clinically advantageous. ${ }^{32-39}$

Rigid interspinous process fixation (ISPF) is one such alternative modality receiving increased consideration as an adjunct to ALIF/LLIF. 1,18-20,23,29,31 Given the favorable proximity of the spinous processes, one can achieve robust sagittal stability while largely preserving the paraspinal muscles, avoiding the posterior neural elements, and conserving the facets. ${ }^{1,18-20,23,29,31,40}$ However, despite such biomechanical and perioperative benefits, reports of midterm and long-term clinical outcomes with ISPF are limited and retrospective in design. ${ }^{41}$

Accordingly, the purpose of this prospective, randomized, controlled, multicenter clinical trial was to assess intraoperative outcomes, patientreported outcomes, radiographic success, concomitant medication usage, neurologic status, and complication profiles in patients receiving singlelevel ALIF/LLIF with supplemental ISPF for degenerative pathology. A control cohort of PSF patients was used; however, the authors emphasize that the primary rationale for having a control group was to marginalize posterior technique selection bias. Given that there is currently considerable heterogeneity in PSF techniques (ie, unilateral vs. bilateral fixation; open vs. minimally invasive access; navigation aided), identification of a pertinent homogenous PSF cohort for direct comparative purposes was challenging. ${ }^{19,39,42-44}$ Accordingly, outcomes with ISPF should be considered primarily within the context of what is clinically advantageous (ie, minimal clinically important difference [MCID] values), with direct comparison to PSF outcomes considered secondarily only when appropriate.

\section{MATERIALS AND METHODS}

\section{Study Design and Reporting}

This was a prospective, randomized, controlled, multicenter clinical trial to compare the outcomes of patients who received ISPF (investigational group) or PSF (control group) as an adjunct to single-level ALIF/LLIF for the treatment of degenerative disc disease and/or spondylolisthesis (<grade 2). The trial was conducted across 9 multidisciplinary spine or neurosurgery centers, with a total of 11 surgeon investigators. Institutional review board approval was obtained at each center (Western IRB, Puyallup, Washington), and informed consent was obtained from all study participants. The study was registered with the ClinicalTrials.gov database with an ID number of NCT01549366. Study protocol adheres to the principles set forth in the US Code of Federal Regulations and the World Medical Association Declaration of Helsinki. Additionally, all study objectives, methods, results, and discussion items are reported in accordance with the CONSORT 2010 guidelines for reporting parallel group randomized trials.

\section{Patient Sample Size, Randomization, and Interim Analyses}

A target randomization ratio of 2:1 ISPF/PSF patients and a target enrollment of 67 patients (44 ISPF and 23 PSF) were desired for study completion. Unequal randomization was used in consideration of (1) a large investigational device sample size for potential secondary subanalyses, and (2) use of PSF in primarily serving to diminish selection bias. Inclusion and exclusion criteria are listed in Table 1. The target sample size assumed a $30 \%$ attrition rate and a goal of at least $80 \%$ power in testing the primary noninferiority hypothesis (see "Primary Study Outcome and Hypothesis"). Parameter values used in the sample size estimation included an $\alpha$ value of 0.05 (type I error), a $\beta$ value of 0.2 (type II error), and a standard deviation assumption of 20 points for ODI score change. An interim analysis was performed when $50 \%$ of patients had reached the primary end point of 12 months to assess whether a sample size adjustment was needed. An early stop for success or futility was allowed if necessary. No such sample size adjustment or early stop was needed.

\section{Randomization Allocation, Concealment, and Implementation}

In accordance with the desired randomization ratio, an allocation sequence was generated by the study sponsor and provided to each study site. Block randomization was used to avoid imbalance in the number of patients assigned to each treatment group within a site. The posterior fixation assignment was then provided to the investigator by the study site manager in a sealed envelope just prior to surgery. Patients were blinded to the instrumentation until after the surgery. 
Table 1. Study inclusion, exclusion, and intraoperative exclusion criteria

Inclusion criteria

Age between 18 and $75 \mathrm{y}$

Scheduled for an elective single-level circumferential lumbar fusion

by means of an anterior or lateral interbody fusion with

supplemental posterior fixation (allowing for the placement of ISPF or PSF)

Diagnosis of primary symptomatic degenerative disc disease and/or spondylolisthesis confirmed with appropriate imaging studies and/ or positive lumbar discography

Note: Degenerative disc disease was defined as back pain of discogenic origin with degeneration of the disc confirmed by history and radiographic studies

ODI v2.1 score $\geq 30 \%$

Failed at least $6 \mathrm{wk}$ of conservative care (nonsurgical) $O R$ has

clinical signs of neurologic deterioration

Signed informed consent form

Exclusion criteria

Previous fusion at the operative level

Spondylolisthesis grade 3 or more

Lytic spondylolisthesis

Incompetent or missing posterior arch at the affected level (eg, complete laminectomy, pars defect)

Requires complete laminectomy at level of surgery

Facet joints at implant level are absent or fractured

Vertebral body compromise or acute fracture at implant level

Body mass index $\geq 40$

Known allergy to titanium

Osteoporosis: Simple Calculated Osteoporosis Risk Estimation (SCORE) $\geq 6 A N D$ dual-energy x-ray absorptiometry (DEXA) Tscore less than -2.5

Paget disease, osteomalacia, or any other metabolic bone disease Use of medications or any drug known to potentially interfere with bone/soft tissue healing (eg, chronic systemic steroids)

Planned use of additional segmental fixation (eg, facet screws)

Planned use of BMP for posterolateral fusion

Unlikely to comply with the follow-up evaluation schedule In the opinion of the investigator, patient has history of chemical substance dependency or significant psychosocial disturbance that may impact the outcome or study participation

Active participation in a clinical trial of another drug or device

Active systemic infection or any other health condition that would preclude surgery

History of invasive malignancy, except if the patient has received treatment and displayed no clinical signs and symptoms for at least $5 \mathrm{y}$

Patient is a prisoner

Pregnant or planning to become pregnant during the length of study participation

Involvement in active litigation related to back problems at the time of screening (this does not apply to litigation in no-fault states)

Direct involvement in the execution of this protocol

Preexisting conditions that could interfere with the evaluation of outcome measures (eg, musculoskeletal, neuromuscular, etc)

Intraoperative exclusion criteria

Intraoperative visualization of a grade 3 or higher spondylolisthesis not previously noted radiographically

Any change in the surgical procedure prior to surgery that violates the inclusion/exclusion criteria (eg, switching to double-level procedure; decision not to perform posterior fixation) Patients with confounding factors inhibiting the posterior placement of Aspen or pedicle screws will automatically receive the opposing fixation device and will be followed as "intent-to-treat"

Abbreviations: PSF, pedicle screw fixation; ISPF, interspinous process fixation; ODI, Oswestry Disability Index; BMP, bone morphogenic protein.

\section{Study Follow-Up}

Patients were evaluated perioperatively and then at 6 weeks ( \pm 14 days), 3 months ( \pm 14 days), 6 months ( \pm 30 days), and 12 months ( \pm 60 days) postoperatively. Final study follow-up is 24 months ( \pm 60 days).

\section{Primary Study Outcome and Hypothesis}

Per study protocol, the primary study end point outcome was change in Oswestry Disability (Low Back) Index (ODI) score from baseline (preoperative) to 12 months. The primary study hypothesis was noninferiority of the ODI score change by the investigational group (ISPF) compared with the control group (PSF). A noninferiority margin of 10 ODI score points was predefined in accordance with previously reported MCID values. ${ }^{45}$ Noninferior design was used, given that PSF is a well-established and efficacious modality for posterior stabilization.

\section{Secondary Study Outcomes and Measurement Tools}

Secondary study measures included intraoperative outcomes, patient-reported outcomes, radiographic success, concomitant medication usage, neurologic status, and complication profiles. Measurement tools included the ODI Questionnaire, the Zurich Claudication Questionnaire (ZCQ), and the 36-Item Short Form Health Survey (SF-36).

\section{Intraoperative Outcomes and Complications}

Operating time, estimated intraoperative blood loss (EBL), fluoroscopic imaging time, and incisions length(s) were collected for each treatment group and stratified by interbody and posterior procedure. Patient length of stay was not reported because a portion of patients received staged interbody and posterior procedures on separate days. All adverse and serious adverse events that occurred intraoperatively or during follow-up were recorded by the investigators. Investigators indicated each event as either "possibly related" or "not related" to the posterior surgery/instrumentation.

\section{Radiographic Outcomes}

Radiographic analysis was performed by an independent, board-certified radiologist (Imaging Endpoints LLC, Scottsdale, Arizona). Radiographic end points included interbody fusion and 
interspinous fusion (ISPF patients only). Interbody fusion (12 months) was evaluated via computed tomography (CT) scans and scored using the Brantigan, Stelfee, Fraser (BSF) criteria: BSF-1, radiographic pseudoarthrosis with loss of intervertebral height with lucency around the implant; BSF2 , radiographic locked pseudoarthrosis with lucency within the cage but solid bone growth into the cage from each vertebral endplate; and BSF-3, radiographic fusion with bony bridges in at least half of the fusion area. $^{46}$ Interspinous fusion (12 months) was assessed in all ISPF patients via CT scan, with fusion success defined as continuous bone bridging spanning the spinous processes within the ISPF device plates, as confirmed on coronal and/or sagittal view.

\section{Concomitant Medication Use and Neurologic Status}

Both concomitant medication use and neurologic status were assessed across treatment group and time. Medication classes considered were narcotics, muscle relaxants, over-the-counter pain medications, nonsteroidal anti-inflammatory drugs, neuroleptics, and antidepressants. Neurologic status was assessed as a function of motor, sensory, deep tendon reflexes, and gait dysfunction.

\section{Statistical Analysis}

Analyses of all patient-reported outcome measures were performed using a linear mixed-model framework that included nested random effects (patients nested within sites) to account for withinsite correlation and for correlation due to repeated measures taken on the patients over time. All statistical tests were performed at the significance level of 0.05 . The primary analysis was a simple comparison between treatment groups of the relative change in ODI scores from baseline to 12 months. Similarly, intraoperative outcomes were analyzed using a linear mixed-model framework that included a random intercept to account for within-site correction. To assess concomitant medication use, a logistic regression generalized estimating equation model with an exchangeable correlation structure was used to compare the odds of using at least 1 medication between treatment groups over time while accounting for within-patient correlation. For neurologic outcomes, a similar generalized estimating equation model was used to compare the odds of having any motor, reflex, sensory, or gait dysfunc-

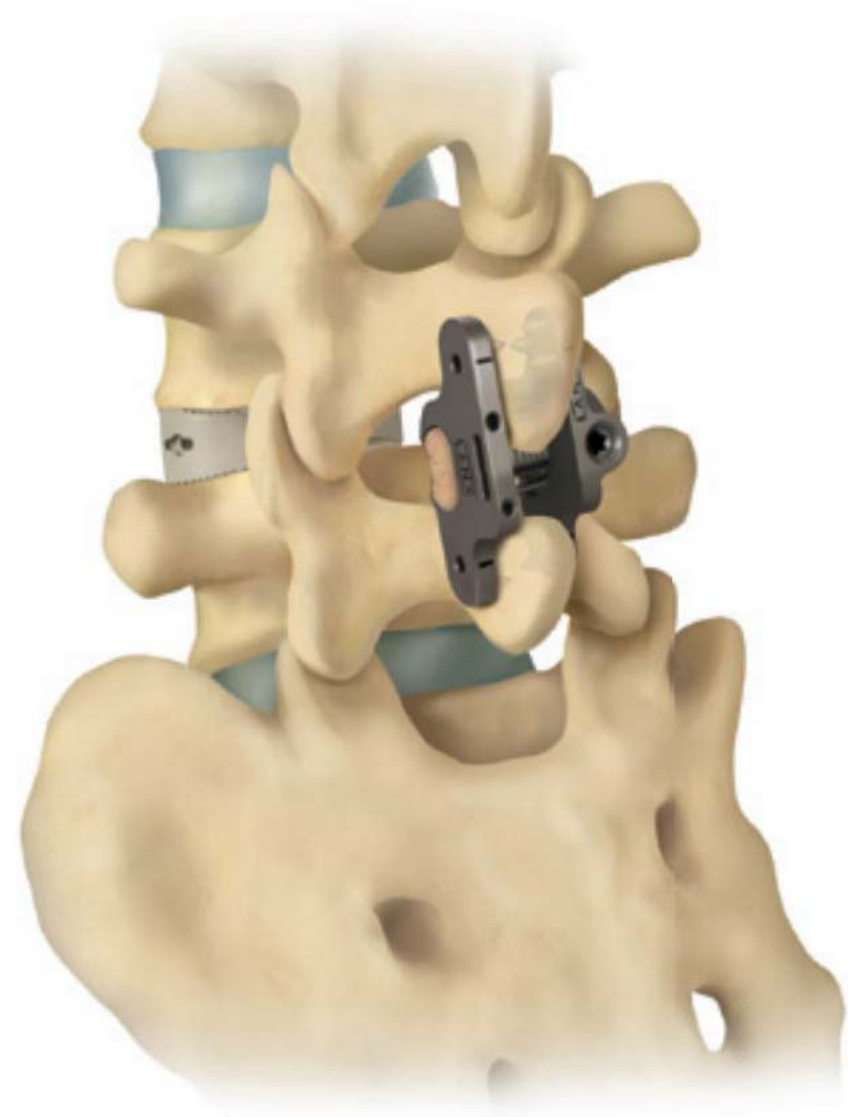

Figure 1. Investigational interspinous process fixation device (Aspen MIS Fusion System).

tion between treatment groups over time. Additionally, a negative binomial generalized linear mixed model for count data was used to model the number of concomitant medications used over time and to determine whether there were differences in the change in the number of medications used between the 2 treatment groups over time. A random intercept was included in the model to account for within-patient correlation. The model was repeated for all individual medication classes. Radiographic outcomes were compared between the 2 treatment groups using Fisher exact test. To compare radiographic outcomes between the treatment groups while adjusting for potential confounders and accounting for within-site correlation, the odds of fusion (BSF-3) were modeled using a generalized estimating equation framework with an exchangeable correlation structure.

\section{Investigational ISPF Device and Technique}

The investigational ISPF device (Aspen MIS Fusion System, Zimmer Biomet Spine, Westminster, 
Table 2. Patient demographic data.

\begin{tabular}{|c|c|c|}
\hline \multirow[b]{2}{*}{ Variable } & \multicolumn{2}{|c|}{ Treatment Groups } \\
\hline & $\begin{array}{c}\text { ISPF } \\
(n=66)\end{array}$ & $\begin{array}{c}\text { PSF } \\
(\mathbf{n}=37)\end{array}$ \\
\hline Age, y, mean (SD) & $50.5(14.0)$ & $52.8(13.2)$ \\
\hline Female sex, n $(\%)$ & $31(47)$ & $26(70.3)$ \\
\hline $\begin{array}{l}\text { Body mass index, } \mathrm{kg} / \mathrm{m}^{2} \text {, mean } \\
\text { (SD) }\end{array}$ & $29.0(5.1)$ & $31.5(5.6)$ \\
\hline \multicolumn{3}{|l|}{ Smoking status, n (\%) } \\
\hline Current & $21(31.8)$ & $13(35.1)$ \\
\hline Former & $10(15.2)$ & $6(16.2)$ \\
\hline Never & $35(53)$ & $18(48.6)$ \\
\hline Work-related injury, $\mathrm{n}(\%)$ & $10(15.2)$ & $4(10.8)$ \\
\hline \multicolumn{3}{|l|}{ On sick leave, n (\%) } \\
\hline Yes & $15(22.7)$ & $2(5.4)$ \\
\hline No & $35(53)$ & $27(73)$ \\
\hline Not employed & $16(24.2)$ & $8(21.6)$ \\
\hline \multicolumn{3}{|l|}{ Diagnosis, n (\%) } \\
\hline Degenerative disc disease & $36(54.5)$ & $16(43.2)$ \\
\hline Spondylolisthesis & $8(12.1)$ & $2(5.4)$ \\
\hline $\begin{array}{l}\text { Degenerative disk disease }+ \\
\text { spondylolisthesis }\end{array}$ & $22(33.3)$ & $19(51.4)$ \\
\hline \multicolumn{3}{|l|}{ Surgical level, n (\%) } \\
\hline L2-3 & $3(4.5)$ & 0 \\
\hline L3-4 & $4(6.1)$ & $3(8.1)$ \\
\hline L4-5 & $38(57.6)$ & $22(59.5)$ \\
\hline L5-S1 & $20(30.3)$ & $12(32.4)$ \\
\hline L5-6 & $1(1.5)$ & 0 \\
\hline \multicolumn{3}{|l|}{ Interbody approach, $\mathrm{n}(\%)$} \\
\hline ALIF & $28(42.4)$ & $17(45.9)$ \\
\hline LLIF & $38(57.6)$ & $20(54.1)$ \\
\hline Anterior or lateral plate & $23(34.8)$ & $13(35.1)$ \\
\hline \multicolumn{3}{|l|}{ Bone graft, $\mathrm{n}(\%)$} \\
\hline Autograft & $20(30.3)$ & $13(35.1)$ \\
\hline Allograft & $43(65.2)$ & $22(59.5)$ \\
\hline DBM & $50(75.8)$ & $28(75.7)$ \\
\hline BMP & $13(19.7)$ & $10(27)$ \\
\hline Synthetic & 0 & $3(8.1)$ \\
\hline
\end{tabular}

Abbreviations: ISPF, interspinous process fixation; PSF, pedicle screw fixation; ALIF, anterior lumbar interbody fusion; LLIF, lateral lumbar interbody fusion; DBM, demineralized bone matrix; BMP, bone morphogenic protein.

Colorado) is a posterior fixation device intended to provide stabilization of segments in the thoracic, lumbar, and sacral spine (T1-S1) to support fusion (Figure 1). It is not indicated for stand-alone use. The device contains 2 titanium alloy plates and is assembled by sliding the lock plate, which possesses a torque-controlled set screw, over the post plate until contact is made with the spinous processes. Once in contact, the device plates are compressed such that the plate fixation spikes become seated within the bones of the respective spinous processes. The set-screw mechanism is then engaged to lock the device in place.

Additionally, the device possesses an open bone graft enclosure to support placement of bone graft material within the interspinous space, has coronal plane angulation capabilities $\left( \pm 10^{\circ}\right)$ of the lock plate to accommodate patient anatomy, and is available in multiple device footprints to adapt to specific levels and anatomy.

\section{Surgical Technique}

Study protocol dictated that intervertebral access could be performed via an isolated anterior (ALIF) or lateral (LLIF) approach. ALIF or LLIF was chosen at the discretion of the investigator in accordance with the investigator's institutional standard of care (SOC); distributions were nearly similar between treatment groups and are shown in Table 2. Intervertebral spacer size (footprint) was dictated by patient anatomy and determined by the surgeon investigator in accordance with SOC. The authors acknowledge that heterogeneity in footprint size is a potential limitation; however, this remains an inherent limitation of any interbody fusion clinical study in which patient anatomy is variable. Additionally, the type of intervertebral spacer and type of biologics used were chosen by the investigator in accordance with the investigator's institutional SOC. No significant differences in intervertebral graft material distribution were observed between cohorts $(P \geq .49$; Table 2$)$. Use of bone morphogenetic proteins (BMPs) was not permitted in the posterior surgery. Distribution of intervertebral BMP use between cohorts was consistent (ISPF, 19.7\%; PSF, 27\%). Statistical analysis of BMP as a potential confounder of ISPF patientreported outcomes (ODI, SF-36, ZCQ) was performed, and no correlation was observed $(P \geq .25)$. A significant correlation was found between BMP use and improved PSF patient-reported outcomes for ODI $(P=.02)$, SF-36 PCS $(P=.05)$, and ZCQ symptom severity $(P=.05)$.

Additional posterior segmental fixation was not permitted; however, anterolateral plating was permitted. Given that use of anterolateral plating is now often commonplace within standard ALIF/ LLIF care, particularly in the case of integrated ALIF/LLIF devices, it was considered clinically relevant within the scope of this study. Use of randomization provided nearly equal distribution of plating between cohorts (ISPF, $34.8 \%$ vs. PSF, $35.1 \%$ ). Statistical controlling of plating use and interbody technique as potential confounders was performed when analyzing both patient-reported ODI and interbody fusion outcomes.

Both open $(67.2 \%)$ and minimally invasive/ percutaneous $(32.4 \%)$ PSF techniques were permitted, as was the use of either unilateral $(45.9 \%)$ or bilateral $(54.1 \%)$ PSF. Polyaxial top-loading pedicle screws possessing appropriate US Food and Drug Administration (FDA) indication were required. All 


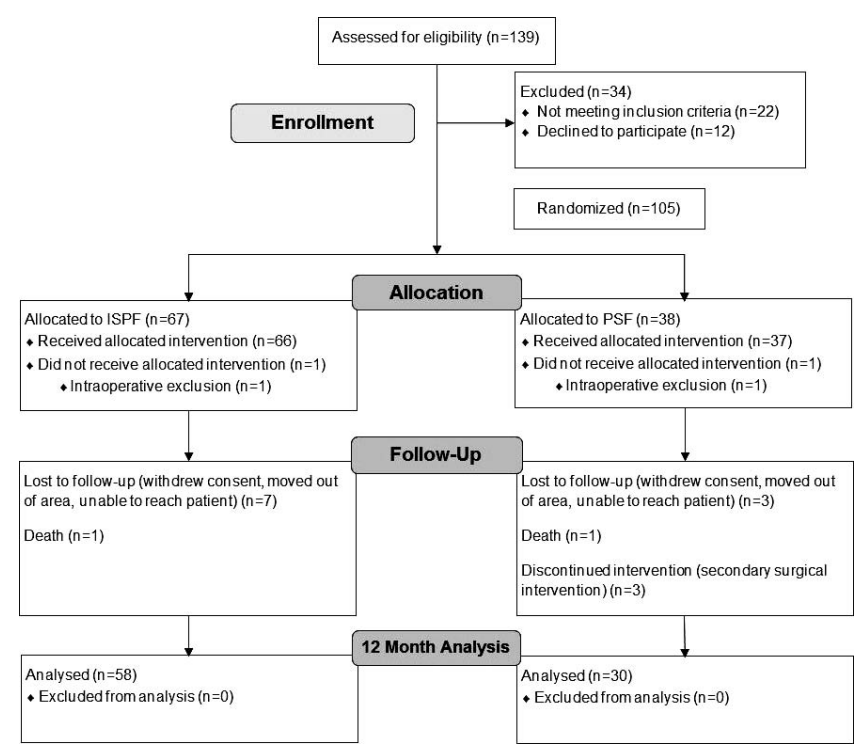

Figure 2. Flow chart reflecting numbers of patients at enrollment, allocation, and follow-up, including reason(s) for exclusion/lost to follow-up.

PSF procedures were performed in accordance with investigator institutional SOC. The authors acknowledge the heterogeneity permitted in the PSF technique; however, such heterogeneity, across multiple sites, is representative of the diverse philosophy around PSF and standard "real world" clinical care. ${ }^{19,39,42-44}$ Furthermore, as previously noted, the authors emphasize that the use of a PSF control group was to minimize posterior technique selection bias. Accordingly, comparison of outcomes between ISPF and PSF groups should be made with caution; instead, emphasis should be placed on ISPF outcomes within the context of MCID values and what is clinically advantageous at large.

ISPF approach and instrumentation were performed according to the device's surgical technique guide. A small midline incision was first made over the index spinous processes; musculature was then incised via a standard midline approach. The spinous processes and lamina were then exposed to the medial border of the facet joints, while the supraspinous ligament was preserved. The interspinous ligament was then pierced as far anteriorly as possible using a dilator. Using a spreader placed within the interspinous space, the appropriate size of the ISPF implant was then determined. The spinous processes were then decorticated using a rasp. The post plate body of the device was placed first, anatomically to the left of the spinous processes, with the barrel portion packed with the
Table 3. Patient intraoperative data: posterior procedure only.

\begin{tabular}{lccc}
\hline & \multicolumn{2}{c}{ Treatment Groups } & \\
\cline { 2 - 3 } Measure & ISPF $(\mathbf{n}=\mathbf{6 6})$ & PSF $(\mathbf{n}=\mathbf{3 7}) *$ & $\boldsymbol{P}$ Value \\
\hline EBL, mL & & & $<.001$ \\
$\quad$ Mean \pm SD & $70.9 \pm 55.3$ & $119.9 \pm 101.9$ & \\
$\quad$ Median & 50.0 & 75.0 & \\
$\quad$ Range & $0-350.0$ & $10.0-500.0$ & \\
Operative time, min & $52.2 \pm 22.8$ & $78.9 \pm 44.0$ & \\
$\quad$ Mean \pm SD & 43.5 & 67.0 & \\
Median & $19.0-135.0$ & $17.0-241.0$ & \\
$\quad$ Range & & & \\
Incision lengths, cm & $5.5 \pm 1.5$ & $7.2 \pm 3.4$ & \\
$\quad$ Mean \pm SD & 5.0 & 7.0 & \\
Median & $2.5-9.0$ & $1.0-15.2$ & \\
$\quad$ Range & & & \\
Fluoroscopy time, s & $10.4 \pm 9.3$ & $57.4 \pm 54.0$ & \\
$\quad \begin{array}{l}\text { Mean } \pm \text { SD } \\
\text { Median }\end{array}$ & 10 & 36.5 & \\
Range & $1.0-51.0$ & $2.5-258.0$ & \\
\hline
\end{tabular}

Abbreviations: ISPF, interspinous process fixation; PSF, pedicle screw fixation; EBL, estimated interoperative blood loss.

* Note: PSF outcomes include those outcomes for all patients regardless of whether they were unilateral/bilateral screws or open/MIS/percutaneous placement. Outcomes should be considered accordingly.

graft material of choice. The matting lock plate was then placed over the post plate on the contralateral side of the spinous processes such that intimate contact was made with bone. The device was placed as anteriorly as possible in order to grip the thicker bone mass at the laminar junction. All surgeons ensured that the device did not protrude above the lumbodorsal fascia and that the fixation spikes effectively engaged the spinous processes prior to final compression. Additional angulation of the plates was performed if necessary. Final plate compression and set-screw tightening were then performed. Final device placement was confirmed via anterior/posterior and lateral fluoroscopic imaging.

\section{RESULTS}

\section{Patient Demographics}

A total of 103 patients (66 ISPF and 37 PSF) were enrolled into the study. Patient follow-up is summarized in the flow diagram in Figure 2. Patient demographic characteristics are summarized in Table 2.

\section{Intraoperative Outcomes}

Intraoperative outcomes are summarized in Table 3. For ISPF patients, mean posterior intraoperative metrics were: blood loss, $70.9 \mathrm{~mL}$; mean operating 


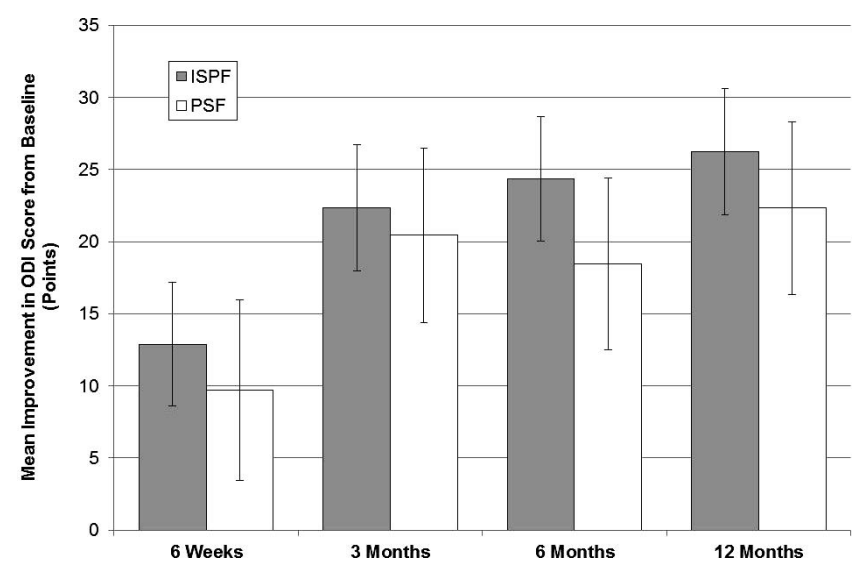

Figure 3. Mean ODI score improvement from baseline at 6 weeks, 3 months, 6 months, and 12 months; no significant differences $(P<.05)$ were observed between cohorts at any time point. Scores at each time point were significantly reduced from baseline for both cohorts $(P<.01)$. Abbreviations: ODI, Oswestry Disability Index; ISPF, interspinous process fixation; PSF, pedicle screw fixation.

time, 52.2 minutes; incision length, $5.5 \mathrm{~cm}$; and fluoroscopic imaging time, 10.4 seconds.

\section{ODI Score}

Mean improvements in ODI score from baseline to 6 weeks, 3 months, 6 months, and 12 months postoperatively were $12.78 \pm 4.12,22.23 \pm 4.23$, $24.01 \pm 4.19$, and $25.97 \pm 4.23$ points, respectively, for ISPF patients (Figure 3). Statistically significant ODI score improvement was achieved in ISPF patients by 6 weeks postoperatively $(P<.01)$ and further maintained out to 12 months $(P<.01)$. Similarly, 6-week, 3-month, 6-month, and 12-month score improvements for PSF patients were $9.23 \pm 6.04,19.92 \pm 5.91,18.69 \pm 5.78$, and $22.38 \pm 5.84$, respectively $(P<.01)$.

Noninferiority $(<10$-point mean difference) of ODI score improvement, relative to baseline, at 12 months was demonstrated by the ISPF group compared with the PSF control group (mean difference, 3.60 points; $95 \%$ confidence interval $[\mathrm{CI}],-3.62$ to 10.81 points; $P=.33$; Figure 3 ). No changes in statistical trends were observed after adjusting for potential confounders (anterolateral plating; interbody technique; $P \geq .329$ ).

\section{ZCQ Scores}

Mean 12-month improvements in ZCQ physical function, symptom severity, and satisfaction scores, relative to baseline, were $0.86 \pm 0.20,1.04 \pm 0.23$, and $1.84 \pm 0.20$, respectively, for ISPF patients. Similarly, 12-month score improvements for PSF patients were $0.76 \pm 0.28,0.99 \pm 0.32$, and
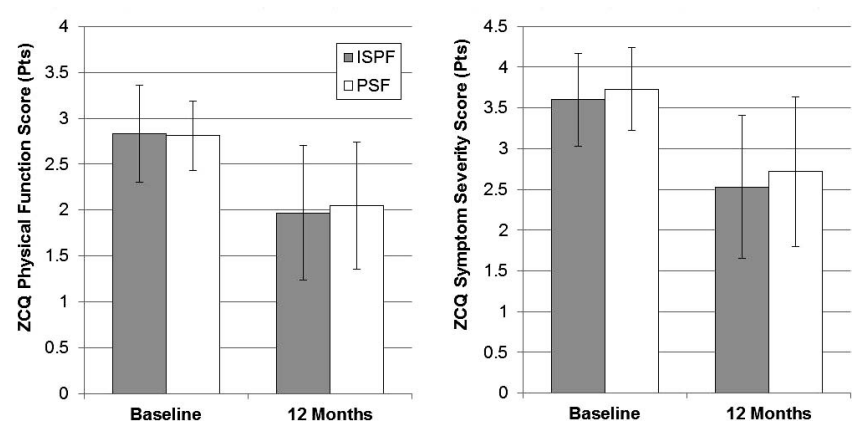

Figure 4. ZCQ physical (left) and ZCQ symptom (right) scores at baseline and 12 months; no significant differences $(P<.05)$ were observed between cohorts at either time point. Scores were significantly reduced from baseline for both cohorts $(P<.01)$. Abbreviations: ZCQ, Zurich Claudation Questionnaire; ISPF, interspinous process fixation; PSF, pedicle screw fixation.

$1.97 \pm 0.27$, respectively. Mean improvement, relative to baseline, for all ZCQ scores, across both cohorts, was statistically significant at 12 months $(P<.01)$. Mean improvement was not significantly different between cohorts for any ZCQ score at 12 months $(P \geq .44$; Figure 4).

\section{SF-36 Scores}

Mean 12-month improvements in SF-36 physical component, mental component, and bodily pain scores, relative to baseline, were $10.87 \pm 2.79$, $9.05 \pm 4.04$, and $31.49 \pm 6.68$, respectively, for ISPF patients. Similarly, 12-month score improvements for PSF patients were 9.10 \pm 3.89 , $6.04 \pm 5.65$, and $24.38 \pm 9.14$, respectively. Mean improvement, relative to baseline, for all SF-36 scores, across both cohorts, was statistically significant at 12 months $(P<.01)$. Mean improvement was not significantly different between cohorts for either SF-36 score at 12 months $(P \geq .22$; Figure 5).

\section{Concomitant Medication Use and Neurologic Status}

The odds of using at least 1 concomitant medication were not significantly associated with either treatment group $(P=.5)$. Additionally, there was not a significant difference in the change in number of medications used over time between treatment groups $(P=.21)$. There was no significant difference in change of odds of motor, reflex, sensory, or gait dysfunction between treatment groups $(P \geq .1)$.

\section{Radiographic Outcomes}

In the ISPF group, the observed distributions of radiographic fusion at 12 months were $45.5 \%$ for 

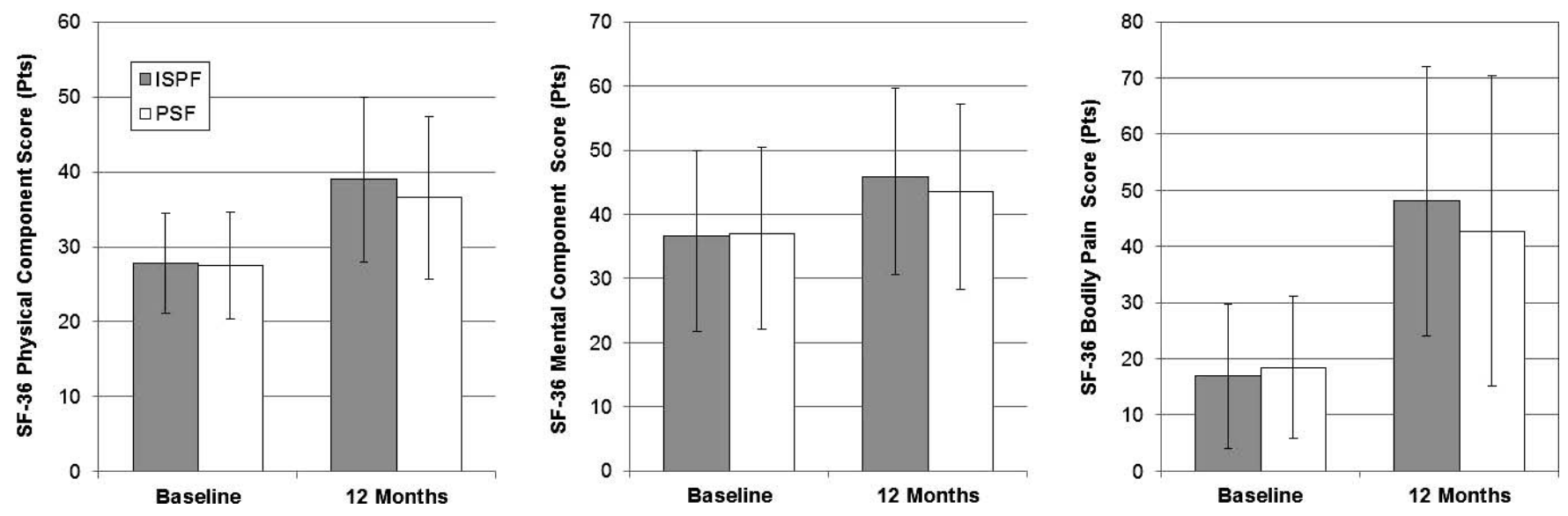

Figure 5. SF-36 physical (left), SF-36 mental (middle), and SF-36 bodily pain (right) scores at baseline and at 12 months; no significant differences $(P<.05)$ were observed between cohorts at either time point. Scores were significantly reduced from baseline for both cohorts $(P<.01)$. Abbreviations: SF-36, 36-Item Short Form Health Survey; ISPF, interspinous process fixation; PSF, pedicle screw fixation.

BSF-3 $(95 \%$ CI, $32.7 \%-59.6 \%), 45.5 \%$ for BSF-2 $(95 \%$ CI, $32.7 \%-59.6 \%)$, and $9.1 \%$ for BSF-1 $(95 \%$ CI, $0.0 \%-23.2 \%)$. In the PSF group, the observed distributions were $50 \%$ for BSF-3 $(95 \%$ CI, 33.3\%$67.8 \%$ ), 50\% for BSF-2 (95\% CI, 33.3\%-67.8\%), and $0 \%$ for BSF-1 $(95 \% \mathrm{CI}, 0.0 \%-17.8 \%)$. There was no significant difference in the distribution of BSF scores between treatment groups at 12 months $(P=.33)$. Additionally, there was no significant difference in the odds of fusion (BSF-3) between treatment groups $(P=.61)$. No changes in statistical trends were observed after adjusting for potential confounders (anterolateral plating; interbody technique; $P \geq .565$ ). Interspinous process fusion was present in $92.7 \%$ of ISPF patients. The 4 patients without observed spinous process fusion all received interbody fusion at the L4-5 level (1 ALIF and 3 LLIF), all achieving a BSF-2 score. All 4 patients demonstrated improvement in their 12-month ODI scores.

\section{Intraoperative and Postoperative Complications}

No posterior device-related complications were observed intraoperatively in either treatment group.
Postoperative complications, both posterior device related and nonrelated, are summarized in Tables 4 and 5 , as indicated by investigator. There was 1 ISPF patient $(1.5 \%)$ and 4 PSF patients $(10.8 \%)$ who required a secondary surgical intervention in possible relation to posterior fixation. All posterior revisions resulted in resolution of symptoms.

\section{DISCUSSION}

The objective of this clinical trial was to assess the efficacy of ISPF in support of ALIF/LLIF. Although the minimally disruptive nature of ISPF is very appealing, it remains largely unknown whether ISPF possesses the structural capabilities to support solid fusion and other long-term clinical successes. This is the first prospective, randomized, controlled, multicenter clinical trial studying the outcomes of ALIF/LLIF with adjunctive ISPF. ${ }^{27}$

Consistent with claims in previous literature, this study substantiated that ISPF can be achieved quickly (mean OR time, 52.5 minutes) and with a diminished operative footprint (mean EBL, 70.9 $\mathrm{mL}$; mean incision length, $5.5 \mathrm{~cm}) .{ }^{38,40,47-50}$ Subse-

Table 4. Posterior device/procedure-related postoperative complications.

\begin{tabular}{|c|c|c|c|c|}
\hline \multirow[b]{2}{*}{ Type } & \multicolumn{2}{|l|}{ ISPF } & \multicolumn{2}{|l|}{ PSF } \\
\hline & Description (n) & $\%(n)$ & Description (n) & $\%(n)$ \\
\hline Hardware & Interbody cage migration (1) & $1.5(1)$ & - & 0 \\
\hline Vertebral & Early evidence of pseudarthrosis (1) & $1.5(1)$ & Adjacent-level vertebral fracture $(1 \dagger)$ & $2.7(1)$ \\
\hline Neuromuscular & Leg or back pain/radiculopathy* $(2,1 \dagger)$ & $4.5(3)$ & Leg or back pain/radiculopathy* $(2,3 \dagger)$ & $13.5(5)$ \\
\hline Total & - & $7.5(5)$ & - & $16.2(6)$ \\
\hline Revisions & - & $1.5(1)$ & - & $10.8(4)$ \\
\hline
\end{tabular}

Abbreviations: ISPF, interspinous process fixation; PSF, pedicle screw fixation.

*Any leg pain, back pain, or radiculopathy that was indicated as new, recurrent, or increased relative to preoperative levels.

$\dagger$ Required secondary surgical intervention. 
Table 5. Summary of cumulative procedure related complications (indicated as NOT posterior device-related).

\begin{tabular}{|c|c|c|c|c|}
\hline \multirow[b]{2}{*}{ Type } & \multicolumn{2}{|l|}{ ISPF } & \multicolumn{2}{|l|}{ PSF } \\
\hline & Description (n) & $\%(n)$ & Description & $\%(n)$ \\
\hline Neuromuscular & $\begin{array}{l}\text { Leg or back pain/radiculopathy* (6); } \\
\text { muscle spasms (1) }\end{array}$ & $10.6(7)$ & $\begin{array}{l}\text { Leg or back pain/radiculopathy* } \\
\text { (4); impaired reflexes (1) }\end{array}$ & $13.5(5)$ \\
\hline Renal & Urinary dysfunction (1) & $1.5(1)$ & Urinary dysfunction (1) & $2.7(1)$ \\
\hline Wound & Interbody incision infection (1) & $1.5(1)$ & Interbody incision pain (1) & $2.7(1)$ \\
\hline Gastrointestinal & Ileus (2); incontinence (1) & $4.5(3)$ & - & 0 \\
\hline Vascular & Dehydration (1) & $1.5(1)$ & - & 0 \\
\hline Respiratory & Pulmonary emboli (1) & $1.5(1)$ & - & 0 \\
\hline Total & - & $21.2(14)$ & - & $18.9(7)$ \\
\hline
\end{tabular}

Abbreviations: ISPF, interspinous process fixation; PSF, pedicle screw fixation.

*Any leg pain, back pain, or radiculopathy that was indicated as new, recurrent, or increased relative to preoperative levels.

quently, in combining both ease of implantation and direct visualization with the ISPF technique, use of fluoroscopic imaging was modest (mean time, 10.4 seconds). Such a trend is contradictory to the traditional philosophy surrounding minimal-access techniques in which increased levels of fluoroscopic guidance are typically required for satisfactory outcomes. Although these intraoperative outcomes with ISPF are not necessarily unexpected, these data provide the most conclusive evidence within the literature to date.

Improvements in patient-reported outcome scores were robust for both the ISPF and PSF cohorts, demonstrating significance from baseline to 12 months across all indices $(P<.01)$. In assessing outcomes early in the postoperative period, mean ODI scores for ISPF patients were reduced relative to baseline by $21.8 \%$ at just 6 weeks $(P<.01)$ and by $40.0 \%$ at 3 months $(P<.01)$. Raw ODI score improvement in ISPF patients at 6 weeks (12.8 points) compared favorably with historical MCID ODI values, with values reported at 5.3-9.5 points at 6-8 weeks. $^{51-53}$ Furthermore, raw ODI score improvement in ISPF patients at 12 months (26.0 points) far exceeded historical MCID ODI values for such follow-up, with reported values ranging from 2.9 to 15.4 points. ${ }^{54-57}$ Adogwa et al., ${ }^{54}$ assessing ALIF and LLIF specifically, reported unique ODI MCID values at 12 months of 9 (ALIF) and 8 (LLIF) points. Raw improvements in SF-36 (PCS, 10.9) and ZCQ (symptom severity, 1.04; physical function, 0.86) scores at 12 months were also comparable within the context of historical MCID values. Reported SF-36 PCS MCID values at 12 months have ranged from 1.26 to 12 points, whereas ZCQ symptom severity and physical function MCID values have been reported at 0.48 and 0.52 points, respectively. ${ }^{54,56-58}$ Lastly, pertaining to the primary study hypothesis of noninfer- iority in 12-month ODI score improvement by ISPF patients, compared with PSF patients, a noninferior difference of 3.6 points was observed $(95 \%$ CI, 10.81 to $-3.62 ; P=.33$ ).

To date, the literature on ISPF remains sparse and heterogeneous. Only Vokshoor et al. $^{49}$ have reported clinical outcomes with the Aspen MIS Fusion System in ALIF or LLIF. However, circumferential fusion patients represented approximately only $6 \%-7 \%$ of the sample population. Additional ISPF constructs assessed included standalone ISPF, ISPF + PSF (no interbody), and ISPF + PSF with interbody. As a collective population, patients reported a visual analog scale score decrease of 3.6 points at 3 months relative to baseline, and they maintained this improvement at 6 and 12 months. Within patients who had received a CT scan, $94 \%$ of all levels demonstrated interspinous fusion. Two patients receiving interbody fusion with ISPF (only) exhibited pseudarthrosis. These outcomes, although limited by variation in cumulative technique, are consistent with those observed in this study, which captured an interspinous fusion rate of $92.7 \%$ and a BSF-1 (pseudarthrosis) rate of $9.1 \%$ at 12 months. However, it should be noted that those patients scoring a BSF-1 remained asymptomatic.

Babu et al. ${ }^{40}$ have also characterized clinical outcomes with the Aspen MIS Fusion System; however, adjunctive application with interbody fusion was limited and access technique not defined. A total of 192 patients (374 levels) were assessed. Constructs evaluated included interbody fusion with ISPF $(n=2)$, interbody fusion with ISPF + PSF $(\mathrm{n}=18)$, ISPF only $(\mathrm{n}=142)$, and ISPF + PSF only $(\mathrm{n}=30)$. Given the heterogeneity in technique, any comparison to the current study is challenging. Regarding complication, no device breakage or dislodgement was observed. Spinous process fracturing was observed in $3.1 \%$ of patients $(n=6)$. 
Three reoperations were performed because of new/ worsening postoperative back and/or leg pain in which a possible relation to ISPF was indicated. These outcomes are consistent with those observed in the current study for ISPF patients. One case of interbody device migration and one case of early evidence of pseudarthrosis were observed. No spinous process fracturing was observed. One revision surgery was performed in the ISPF group in which a possible relationship to the device was indicated $(1.5 \%)$.

A key consideration of both Vokshoor et al. ${ }^{49}$ and the current study was that of interspinous fusion as facilitated by the Aspen MIS Fusion device. Unlike traditional posterior screw techniques, as well as most ISPF devices, the Aspen MIS Fusion device possesses a bone graft barrel specifically designed to facilitate fusion within the interspinous space in a controlled fashion. Although posterior bone grafting is certainly possible with traditional fixation techniques, such devices often require expanded access/visualization and use anatomic confinement for graft placement. Accordingly, these techniques lend to increased morbidity/ demand and are susceptible to graft migration. Furthermore, the ability to achieve robust interspinous fusion $(92.7 \%-94 \%)$ with the Aspen MIS Fusion device means that posterior stability is increasing postoperatively. ${ }^{49}$ These phenomena are crucial to consider within the context of biomechanical stability and risk of spinous process fracturing. Much debate has been made as to whether ISPF can provide sufficient stability in support of interbody fusion, with several in vitro studies characterizing the technology against PSF. ${ }^{18-20,23,29,31}$ However, although baseline rigidity between techniques may favor bilateral PSF in most cases, the additional rigidity offered via interspinous fusion must be considered as subsequent stability not accounted for at baseline. Additionally, spinous process fracturing, although not observed in this study, is often considered a potential risk of ISPF use. $^{41}$ The ability to augment the mechanical integrity of the spinous processes via continuous fusion within the interspinous space may help further diminish fracture likelihood. Assessment of ISPF fracture rates in patients with and without interspinous fusion may be warranted in further substantiating such extrapolation. Secondarily, it was found in this study that use of BMP within the intervertebral space had no impact on quality of patient-reported outcomes in ISPF patients $(P \geq .25)$. However, a significant correlation was found between BMP use and improved PSF patientreported outcomes (ODI, SF-36 PCS, and ZCQ symptom severity; $P \leq .05)$. Longer-term follow-up is needed to better understand this trend; however, it does suggest that robust interspinous fusion may be a leveling factor in the correlation of intervertebral fusion quality and patient outcome at 12 months. Furthermore, it can be inferred from this trend that BMP use is not compensating for a potentially "weaker" ISPF construct with respect to patient outcome quality at 12 months. Fusion analysis at 24 months will be more telling in understanding the relationship between BMP use and quality of outcomes.

Lastly, a key consideration of ISPF is the ability to preserve the facets and better maintain the inherent stability of adjacent levels as well as promote physiologic load sharing. As previously examined in the literature, facet violation can lead to decreased stability in adjacent segments, as well as predispose to accelerated facet joint arthropathy and degeneration. ${ }^{5-61} \mathrm{~A}$ technical characteristic of PSF placement involves risk of facet violation, with reported rates ranging from $11 \%$ to $100 \%$ depending on access technique. ${ }^{62}$ Although this study did not explicitly quantify facet joint integrity postoperatively, no adverse events were reported in which adjacent-level pathology was indicated. One PSF patient exhibited an adjacent-level vertebral fracture. Furthermore, 2 studies of ISPF (not specifically the Aspen MIS Fusion System) with interbody fusion have demonstrated diminished rates of adjacent segment disease (ASD) and facet degeneration. Kim et al., ${ }^{63}$ evaluating both ISPF and PSF as adjuncts to posterior lumbar interbody fusion, reported incidence of ASD at a minimum of 12 months' follow-up of $12.5 \%$ (ISPF) and $36.1 \%$ (PSF) $(P=.029)$. Similarly, Chen and Chen, ${ }^{64}$ also evaluating ISPF in posterior lumbar interbody fusion, reported no incidence of ASD at 12 months. Diminished rates of ASD are also important to consider within the context of local lordosis. A perceived limitation of ISPF is that it cannot afford the same degree of lordotic correction as PSF and could potentially introduce local kyphosis by distracting the interspinous space. However, although PSF may provide greater mechanical leverage to induce lordosis, this may incur facet violation and again predispose to ASD. Although no 
definitive evidence exists differentiating PSF as a more effective posterior fixation modality with respect to sagittal balance maintenance, the diminished rates of ASD with ISPF provide compelling evidence that ISPF, despite the perceived limitations, can provide effective sagittal correction and preservation. Furthermore, the use of large anterior/ lateral lordotic angled intervertebral cages can provide a source lordosis induction without necessitating significant induction via posterior manipulation. The authors do acknowledge that local kyphosis may occur if the ISPF device is oversized or inappropriately placed within the interspinous space; however, proper device trialing and placement mitigates this risk.

The authors acknowledge that limitations did exist within this study, including the heterogeneity of PSF techniques. However, as emphasized, the use of a PSF control group significantly marginalizes any posterior technique selection bias. Accordingly, outcomes with ISPF should be considered within the context of what is clinically meaningful, with comparison to PSF outcomes contemplated only when appropriate. Heterogeneity also existed in the use of anterolateral plating; however, use of randomization and statistical controlling demonstrated a marginalized effect. Furthermore, standardization of concomitant medication(s) and intraoperative use of biologics were not performed; however, randomization of cohorts resulted in comparable distributions between cohorts that align with routine standard of care.

\section{CONCLUSION}

Through 12 months of follow-up, adjunctive ISPF demonstrated efficient, minimally disruptive posterior stabilization in ALIF/LLIF, supporting significant improvement in patient-reported outcomes and facilitating robust posterior fusion. Continued, rigorous assessment of study cohorts out to 24 months of follow-up will further the understanding of safety and efficacy of ISPF in single-level circumferential spinal arthrodesis.

\section{ACKNOWLEDGMENTS}

Study was initiated and funded by Zimmer Biomet Spine Inc (Clinicaltrials.gov ID: NCT01549366). The device(s)/drug(s) used in this study is/are FDA approved or approved by a corresponding national agency. The Aspen MIS Fusion System (Zimmer
Biomet Spine) is indicated by the FDA for use as an adjunct to interbody fusion. This study evaluates the device in this application only.

\section{REFERENCES}

1. Berjano P, Langella F, Damilano M, et al. Fusion rate following extreme lateral lumbar interbody fusion. Eur Spine J. 2015;24(suppl 3):369-371.

2. Härtl R, Joeris A, Mcguire RA. Comparison of the safety outcomes between two surgical approaches for anterior lumbar fusion surgery: anterior lumbar interbody fusion (ALIF) and extreme lateral interbody fusion (ELIF). Eur Spine J. 2016;25(5):1484-1521.

3. Joseph JR, Smith BW, La Marca F, Park P. Comparison of complication rates of minimally invasive transforaminal lumbar interbody fusion and lateral lumbar interbody fusion: a systematic review of the literature. Neurosurg Focus. 2015;39(4):E4.

4. Kim JS, Kim DH, Lee SH, et al. Comparison study of the instrumented circumferential fusion with instrumented anterior lumbar interbody fusion as a surgical procedure for adult low-grade isthmic spondylolisthesis. World Neurosurg. 2010;73(5):565-571.

5. Lehmen JA, Gerber EJ. MIS lateral spine surgery: a systematic literature review of complications, outcomes, and economics. Eur Spine J. 2015;24(suppl 3):287-313.

6. Madan SS, Boeree NR. Comparison of instrumented anterior interbody fusion with instrumented circumferential lumbar fusion. Eur Spine J. 2003;12(6):567-575.

7. Malham GM, Parker RM, Blecher CM, Chow FY, Seex KA. Choice of approach does not affect clinical and radiologic outcomes: a comparative cohort of patients having anterior lumbar interbody fusion and patients having lateral lumbar interbody fusion at 24 months. Global Spine J. 2016;6(5):472481.

8. Min JH, Jang JS, Lee SH. Comparison of anterior- and posterior-approach instrumented lumbar interbody fusion for spondylolisthesis. J Neurosurg Spine. 2007;7(1):21-26.

9. Pawar AY, Hughes AP, Sama AA, Girardi FP, Lebl DR, Cammisa FP. A comparative study of lateral lumbar interbody fusion and posterior lumbar interbody fusion in degenerative lumbar spondylolisthesis. Asian Spine $J$. 2015;9(5):668-674.

10. Phan K, Thayaparan GK, Mobbs RJ. Anterior lumbar interbody fusion versus transforaminal lumbar interbody fusion-systematic review and meta-analysis. $\mathrm{Br} J$ Neurosurg. 2015;29(5):705-711.

11. Sembrano JN, Tohmeh A, Isaacs R. Two-year comparative outcomes of MIS lateral and MIS transforaminal interbody fusion in the treatment of degenerative spondylolisthesis, part I: clinical findings. Spine (Phila $\mathrm{Pa}$ 1976). 2016;41(suppl 8):S123-S132.

12. Winder MJ, Gambhir S. Comparison of ALIF vs. XLIF for L4/5 interbody fusion: pros, cons, and literature review. J Spine Surg. 2016;2(1):2-8.

13. Ahmadian A, Bach K, Bolinger B, et al. Stand-alone minimally invasive lateral lumbar interbody fusion: multicenter clinical outcomes. J Clin Neurosci. 2015;22(4):740-746. 
14. Basra S, Bucklen B, Muzumdar A, Khalil S, Gudipally M. A novel lateral lumbar integrated plate-spacer interbody implant: in vitro biomechanical analysis. Spine $J$. 2015;15(2):322-328.

15. Bess R, Cornwall G, Vance R, Bachus K, Brodke D. In: Goodrich JA, Volcan IJ, eds. Biomechanics of lateral arthrodesis. eXtreme Lateral Interbody Fusion (XLIF). St Louis, Mo: Quality Medical Publishing; 2008:31-40.

16. Cappuccino A, Cornwall GB, Turner AW, et al. Biomechanical analysis and review of lateral lumbar fusion constructs. Spine. 2010;35(26 suppl):S361-S367.

17. Chin KR, Newcomb AG, Reis MT, et al. Biomechanics of posterior instrumentation in L1-L3 lateral interbody fusion: pedicle screw rod construct vs. transfacet pedicle screws. Clin Biomech (Bristol, Avon). 2016;31:59-64.

18. Doulgeris JJ, Aghayev K, Gonzalez-Blohm SA, Lee WE, Vrionis FD. Biomechanical comparison of an interspinous fusion device and bilateral pedicle screw system as additional fixation for lateral lumbar interbody fusion. Clin Biomech (Bristol, Avon). 2015;30(2):205-210.

19. Fogel GR, Parikh R, Ryu S, Turner AW. Biomechanics of lateral lumbar interbody fusion constructs with lateral and posterior plate fixation: laboratory investigation. $J$ Neurosurg Spine. 2014;20(3):291-297.

20. Fogel GR, Turner AW, Dooley Z, Cornwall GB, Biomechanical stability of lateral interbody implants and supplemental fixation in a cadaveric degenerative spondylolisthesis model. Spine (Phila Pa 1976). 2014;39(19):E1138-E1146.

21. Gonzalez-Blohm SA, Doulgeris JJ, Aghayev K, Lee WE, Laun J, Vrionis FD. In vitro evaluation of a lateral expandable cage and its comparison with a static device for lumbar interbody fusion: a biomechanical investigation. $J$ Neurosurg Spine. 2014;20(4):387-395.

22. Hartensuer R, Riesenbeck O, Schulze $M$, et al. Biomechanical evaluation of the Facet Wedge: a refined technique for facet fixation. Eur Spine J. 2014;23(11):23212329.

23. Karahalios DG, Kaibara T, Porter RW, et al. Biomechanics of a lumbar interspinous anchor with anterior lumbar interbody fusion. J Neurosurg Spine. 2010;12(4):372380.

24. Kretzer RM, Molina $\mathrm{C}, \mathrm{Hu} \mathrm{N}$, et al. A comparative biomechanical analysis of stand alone versus facet screw and pedicle screw augmented lateral interbody arthrodesis: an in vitro human cadaveric model. Clin Spine Surg. 2016;29(7):E336-E343.

25. Laws CJ, Coughlin DG, Lotz JC, Serhan HA, Hu SS. Direct lateral approach to lumbar fusion is a biomechanically equivalent alternative to the anterior approach: an in vitro study. Spine. 2012;37(10):819-825.

26. Mantell M, Cyriac M, Haines CM, Gudipally M, O'Brien JR. Biomechanical analysis of an expandable lateral cage and a static transforaminal lumbar interbody fusion cage with posterior instrumentation in an in vitro spondylolisthesis model. J Neurosurg Spine. 2016;24(1):32-38.

27. Nayak AN, Gutierrez S, Billys JB, Santoni BG, Castellvi AE. Biomechanics of lateral plate and pedicle screw constructs in lumbar spines instrumented at two levels with laterally placed interbody cages. Spine J. 2013;13(10):13311338 .

28. Pimenta L, Turner AW, Dooley ZA, Parikh RD,
Peterson MD. Biomechanics of lateral interbody spacers: going wider for going stiffer. Scientific WorldJournal. 2012;2012:381814.

29. Reis MT, Reyes PM, Bse, et al. Biomechanical evaluation of lateral lumbar interbody fusion with secondary augmentation. J Neurosurg Spine. 2016;25(6):720-726.

30. Udby PM, Bech-azeddine R. Clinical outcome of stand-alone ALIF compared to posterior instrumentation for degenerative disc disease: a pilot study and a literature review. Clin Neurol Neurosurg. 2015;133:64-69.

31. Yeager MS, Dupre DA, Cook DJ, Oh MY, Altman DT, Cheng BC. Anterior lumbar interbody fusion with integrated fixation and adjunctive posterior stabilization: a comparative biomechanical analysis. Clin Biomech (Bristol, Avon). 2015;30(8):769-774.

32. Goldstein CL, Macwan K, Sundararajan K, Rampersaud YR. Perioperative outcomes and adverse events of minimally invasive versus open posterior lumbar fusion: metaanalysis and systematic review. J Neurosurg Spine. 2016;24:416427.

33. Hicks JM, Singla A, Shen FH, Arlet V. Complications of pedicle screw fixation in scoliosis surgery: a systematic review. Spine. 2010;35(11):E465-E470.

34. Nasser R, Yadla S, Maltenfort MG, et al. Complications in spine surgery. J Neurosurg Spine. 2010;13(2):144-157.

35. Park JY, Chin DK, Cho YE. Accelerated L5-S1 segment degeneration after spinal fusion on and above L4-5: minimum 4-year follow-up results. J Korean Neurosurg Soc. 2009;45(2):81-84.

36. Park P, Garton HJ, Gala VC, Hoff JT, McGillicuddy JE. Adjacent segment disease after lumbar or lumbosacral fusion: review of the literature. Spine. 2004;29(17):1938-1944.

37. Rampersaud YR, Moro ER, Neary MA, et al. Intraoperative adverse events and related postoperative complications in spine surgery: implications for enhancing patient safety founded on evidence-based protocols. Spine. 2006;31(13):1503-1510.

38. Sclafani JA, Kim CW. Complications associated with the initial learning curve of minimally invasive spine surgery: a systematic review. Clin Orthop Relat Res. 2014;472(6):17111717.

39. Shin BJ, James AR, Njoku IU, Härtl R. Pedicle screw navigation: a systematic review and meta-analysis of perforation risk for computer-navigated versus freehand insertion. $J$ Neurosurg Spine. 2012;17(2):113-122.

40. Babu R, Gottfried ON, Stevenson JC. Outcomes and complications following spinous process fixation: a single-center analysis of 192 cases. Surg Technol Int. 2013;23:283-290.

41. Lopez AJ, Scheer JK, Dahdaleh NS, Patel AA, Smith ZA. Lumbar spinous process fixation and fusion: a systematic review and critical analysis of an emerging spinal technology. Clin Spine Surg. 2017;30(9):E1279-E1288.

42. Cao Y, Chen Z, Jiang C, Wan S, Jiang X, Feng Z. The combined use of unilateral pedicle screw and contralateral facet joint screw fixation in transforaminal lumbar interbody fusion. Eur Spine J. 2015;24(11):2607-2613.

43. Phan K, Leung V, Scherman DB, Tan AR, Rao PJ, Mobbs RJ. Bilateral versus unilateral instrumentation in spinal surgery: systematic review and trial sequential analysis of prospective studies. J Clin Neurosci. 2016;30:15-23.

44. Xiao SW, Jiang H, Yang LJ, Xiao ZM. Comparison of unilateral versus bilateral pedicle screw fixation with cage fusion 
in degenerative lumbar diseases: a meta-analysis. Eur Spine J. 2015;24(4):764-774.

45. Hägg $\mathrm{O}$, Fritzell $\mathrm{P}$, Nordwall $\mathrm{A}$. The clinical importance of changes in outcome scores after treatment for chronic low back pain. Eur Spine J. 2003;12(1):12-20.

46. Fogel GR, Toohey JS, Neidre A, Brantigan JW. Fusion assessment of posterior lumbar interbody fusion using radiolucent cages: x-ray films and helical computed tomography scans compared with surgical exploration of fusion. Spine $J$. 2008;8(4):570-577.

47. Villavicencio AT, Nelson EL, Mason A, Rajpal S, Burneikiene S. Preliminary results on feasibility of outpatient instrumented transforaminal lumbar interbody fusion. J Spinal Disord Tech. 2013;26(6):298-304.

48. Villavicencio AT, Serxner BJ, Mason A, et al. Unilateral and bilateral pedicle screw fixation in transforaminal lumbar interbody fusion: radiographic and clinical analysis. World Neurosurg. 2015;83(4):553-559.

49. Vokshoor A, Khurana S, Wilson D, Filsinger P. Clinical and radiographic outcomes after spinous process fixation and posterior fusion in an elderly cohort. Surg Technol Int. 2014;25:271-276.

50. Wang JC, Haid RW Jr, Miller JS, Robinson JC. Comparison of CD HORIZON SPIRE spinous process plate stabilization and pedicle screw fixation after anterior lumbar interbody fusion: invited submission from the Joint Section Meeting on Disorders of the Spine and Peripheral Nerves, March 2005. J Neurosurg Spine. 2006;4(2):132-136.

51. Cleland JA, Whitman JM, Houser JL, Wainner RS, Childs JD. Psychometric properties of selected tests in patients with lumbar spinal stenosis. Spine J. 2012;12(10):921-931.

52. Ma C, Wu S, Xiao L, Xue Y. Responsiveness of the Chinese version of the Oswestry disability index in patients with chronic low back pain. Eur Spine J. 2011;20(3):475-481.

53. Monticone M, Baiardi P, Vanti C, et al. Responsiveness of the Oswestry Disability Index and the Roland Morris Disability Questionnaire in Italian subjects with sub-acute and chronic low back pain. Eur Spine J. 2012;21(1):122-129.

54. Adogwa O, Elsamadicy AA, Han JL, Cheng J, Karikari I, Bagley CA. Do measures of surgical effectiveness at 1 year after lumbar spine surgery accurately predict 2-year outcomes? J Neurosurg Spine. 2016;25(6):689-696.

55. Carreon LY, Bratcher KR, Canan CE, Burke LO, Djurasovic M, Glassman SD. Differentiating minimum clinically important difference for primary and revision lumbar fusion surgeries. J Neurosurg Spine. 2013;18(1):102-106.

56. Copay AG, Glassman SD, Subach BR, Berven S, Schuler TC, Carreon LY. Minimum clinically important difference in lumbar spine surgery patients: a choice of methods using the Oswestry Disability Index, Medical Outcomes Study questionnaire Short Form 36, and pain scales. Spine $J$. 2008;8(6):968-974.

57. Glassman SD, Copay AG, Berven SH, Polly DW, Subach BR, Carreon LY. Defining substantial clinical benefit following lumbar spine arthrodesis. J Bone Joint Surg Am. 2008;90(9):1839-1847.

58. Van Meirhaeghe J, Fransen P, Morelli D, et al. Clinical evaluation of the preliminary safety and effectiveness of a minimally invasive interspinous process device APERIUS $\left({ }^{\circledR}\right)$ in degenerative lumbar spinal stenosis with symptomatic neurogenic intermittent claudication. Eur Spine J. 2012;21(12):25652572.

59. Cardoso MJ, Dmitriev AE, Helgeson M, Lehman RA, Kuklo TR, Rosner MK. Does superior-segment facet violation or laminectomy destabilize the adjacent level in lumbar transpedicular fixation?: an in vitro human cadaveric assessment. Spine (Phila Pa 1976). 2008;33(26):2868-2873.

60. Kim HJ, Chun HJ, Kang KT, et al. The biomechanical effect of pedicle screws' insertion angle and position on the superior adjacent segment in 1 segment lumbar fusion. Spine (Phila Pa 1976). 2012;37(19):1637-1644.

61. Park P, Garton HJ, Gala VC, Hoff JT, McGillicuddy JE. Adjacent segment disease after lumbar or lumbosacral fusion: review of the literature. Spine (Phila $\mathrm{Pa}$ 1976). 2004;29(17):1938-1944.

62. Babu R, Park JG, Mehta AI, et al. Comparison of superior-level facet joint violations during open and percutaneous pedicle screw placement. Neurosurgery. 2012;71(5):962970 .

63. Kim HJ, Bak KH, Chun HJ, Oh SJ, Kang TH, Yang MS. Posterior interspinous fusion device for one-level fusion in degenerative lumbar spine disease: comparison with pedicle screw fixation-preliminary report of at least one year follow up. J Korean Neurosurg Soc. 2012;52(4):359-364.

64. Chen MH, Chen JY. A novel nonpedicular screw-based fixation in lumbar spondylolisthesis. Biomed Res Int. 2017;2017:5619350.

Disclosures and COI: Institutional review board approval was obtained at each center (Western IRB, Puyallup, Washington), and informed consent was obtained from all study participants. R.P., R.D., C.H., B.S., A.C., S.M., C.M., and K.K. have received financial compensation from the study funding source with respect to consultancy (hourly). B.V., K.M., and C.F. are employees (salary) of the study funding source. No authors have a direct financial relationship with the investigational device (ie, royalties, design surgeon).

Corresponding Author: Kee Kim, MD, University of California Davis Health System, 3301 C Street, Sacramento, CA 95816. Phone: (916) 734-7463; Email: kdkim@ucdavis.edu.

\section{Published 3 August 2018}

This manuscript is generously published free of charge by ISASS, the International Society for the Advancement of Spine Surgery. Copyright (C) 2018 ISASS. To see more or order reprints or permissions, see http://ijssurgery.com. 